\title{
A Study of the First-Order Continuous-Time Bilinear Processes Driven by Fractional Brownian Motion
}

\author{
Abdelouahab Bibi ${ }^{1}$, and Fateh Merahi ${ }^{2, *}$ \\ ${ }^{1}$ Department of Mathematics, UMC(1), Constantine, Algeria \\ ${ }^{2}$ Department of Mathematics, UMC(1), Constantine, Algeria
}

\section{ARTICLE INFO}

\section{Article History}

Received 18 Mar 2017

Accepted 15 Nov 2017

\section{Keywords}

Continuous-time bilinear process Fractional movement Brownian Spectral representation Itồs solution

Long memory property.

2000 Mathematics Subject Classification

22E46, 53C35, 57S20

\section{ABSTRACT}

The continuous-time bilinear $(C O B L)$ process has been used to model non linear and/or non Gaussian datasets. In this paper, the first-order continuous-time bilinear $\operatorname{COBL}(1,1)$ model driven by a fractional Brownian motion ( $f B m$ for short) process is presented. The use of $f B m$ processes with certain Hurst parameter permits to obtain a much richer class of possibly long-range dependent property which are frequently observed in financial econometrics, and thus can be used as a power tool for modelling irregularly series having memory. So, the existence of Itôs solutions and there chaotic spectral representations for time-varying $C O B L(1,1)$ processes driven by $f B m$ are studied. The second-order properties of such solutions are analyzed and the long-range dependency property are studied.

\section{COBL(1,1) DRIVEN BY FRACTIONAL BROWNIAN MOTION}

In discrete-time series analysis, the assumption of linearity and/or Gaussianity is frequently made. Unfortunately these assumption lead to models that fail to capture certain phenomena commonly observed in practice such as limit cycles, asymmetric distribution, leptokurtosis, etc. Motived by these deficiencies, non linear parametric modelling of time series has attracted considerable attention in recent years. Indeed, one of the most useful class of non-linear time series models is the bilinear specification obtained by adding to an Autoregressive moving average (ARMA) model one or more interaction components between the observed series and the innovations. However, it is observed that these models are not be able to give full information about some datasets exhibit unequally spaced observations and hence the resort to a continuous-time version is crucial. So, in this paper we consider a continuous-time bilinear (COBL) processes $(X(t))_{t \in \mathbb{R}}$ defined on some complete probability space $(\Omega, \mathscr{A}, P)$ equipped with a filtration $\left(\mathscr{A}_{t}\right)_{t \geq 0}$ and subjected to be a solution of the following affine time-varying stochastic differential equation $(S D E)$

$$
d X(t)=(\alpha(t) X(t)+\mu(t)) d t+(\beta(t)+\gamma(t) X(t)) d W^{h}(t), t \geq t_{0}, X\left(t_{0}\right)=X_{0}
$$

denoted hereafter $\operatorname{COBL}(1,1)$. The parameters $\alpha(t), \mu(t), \gamma(t)$ and $\beta(t)$ are differentiable complex deterministic functions subject to the following assumption

\section{Condition 1}

A1 For all $T>t_{0}, \int_{t_{o}}^{T}|\alpha(t)| d t<\infty, \int_{t_{0}}^{T}|\mu(t)| d t<\infty, \int_{t_{0}}^{T}|\gamma(t)|^{2} d t<\infty \cdot \int_{t_{0}}^{T}|\beta(t)|^{2} d t<\infty$.

A2 $\alpha(t), \mu(t), \beta(t) \in \mathbb{C}$ and $\mathfrak{R} e(\gamma(t))=0$ and $\mathfrak{R} e\{\alpha(t)\}<0$, for all $t \geq t_{0}$.

In Eq. (1) $\left(W^{h}(t)\right)_{t \in \mathbb{R}}$ is a real $f B m$ with Hurst parameter $h \in 0, \frac{1}{2}$ defined on a basic given filtered stochastic probability space $\left(\Omega, \mathscr{A},\left(\mathscr{A}_{t}\right)_{t \geq 0}, P\right)$, its covariance kernel is $\operatorname{Cov}\left(W^{h}(t), W^{h}(s)\right)=\frac{\kappa(h)}{2}\left(|t|^{2 h+1}+|s|^{2 h+1}-|t-s|^{2 h+1}\right)$, for all $t, s \geq 0$, where $\kappa(h)=$ 
$\frac{\Gamma(1-2 h)}{h(2 h+1) \pi} \cos \left(\frac{\pi}{2}(1-2 h)\right)$ and admits a spectral representation $W^{h}(t)=\int_{\mathbb{R}} \phi_{t}(\lambda)(i \lambda)^{-h} d Z(\lambda)$ where $\phi_{t}(\lambda)=\frac{e^{i t \lambda}-1}{i \lambda}$ and $d Z($.$) is a$ complex-valued Gaussian spectral measure defined on $(\Omega, \mathcal{A}, P)$ with zero mean, variance $E\left\{|d Z(\lambda)|^{2}\right\}=d G(\lambda)=\frac{d \lambda}{2 \pi}$ and where the principal value of $\frac{1}{2 \pi} \int_{\mathbb{R}} \phi_{t}(\lambda) d(\lambda)$ is 0 . Note that the initial state $X\left(t_{0}\right)$ is a random variable, defined on the same probability space $(\Omega, \mathscr{A}, P)$ independent of $\sigma\left(W(t), t_{0} \leq t \leq T\right)$ such that $E\left\{X\left(t_{0}\right)\right\}=m\left(t_{0}\right)$ and $\operatorname{Var}\left\{X\left(t_{0}\right)\right\}=R\left(t_{0}\right)<+\infty$.

It is well known that if $h=0$, then the corresponding $f B m$ reduces to the usual Brownian motion, otherwise, $\left(W^{h}(t)\right)_{t>0}$ is neither a Markovian nor a semimartingales processes and hence the usual calculus cannot be used, so a different calculus is required. This non Markovian processes have not an independent stationary increments and are well suited for modelling data exhibiting a long-range dependency. For an in-depth detailed mathematical framework of the pertinent properties of $f B m$, we refer the reader to Mishura [1] and the references therein.

The SDE Eq. (1) is called time-invariant when $\alpha(t), \mu(t), \gamma(t)$ are complex deterministic constant functions, i.e., there is some constants complex $\alpha, \mu, \gamma$ such that $\alpha(t)=\alpha, \mu(t)=\mu, \gamma(t)=\gamma$ and for all $t$.

The SDE Eq. (1) encompasses many commonly used models in the literature. Some examples among others are

1. First-order continuous-time autoregressive processes (CAR(1) for short): This classes of $S D E$ may be obtained by assuming $\gamma(t)=0$ for all $t$ (see [2] and the reference therein).

2. Gaussian Ornstein-Uhlenbeck $(O U)$ process: The Gaussian $O U$ process is defined as $d X(t)=(\mu(t)-\alpha(t) X(t)) d t+\beta(t) d W^{(h)}(t)$, with $\beta(t)>0$ for all $t \geq 0$. So it can be obtained from SDE Eq. (1) by assuming $\gamma(t)=0$ for all $t$ (see [3] and the reference therein).

3. Nelson's diffusion process: In the diffusion process of Nelson (see [4], Chapter 2), the time-varying volatility process may be defined as the second-order solution process $(V(t))_{t \geq 0}$ of $d V(t)=\lambda(t)(\mu(t)-V(t)) d t+\gamma(t) V(t) d W^{(h)}(t)$ in which $\lambda(t), \mu(t)$ and $\gamma(t)$ are positive deterministic functions. This $S D E$ can be obtained easily from Eq. (1).

4. Geometric Brownian motion $(G B M)$ : This class of processes is defined as a $\mathbb{R}$-valued solution process $(X(t))_{t \geq 0}$ of $d X(t)=$ $\alpha(t) X(t) d t+\gamma(t) X(t) d W^{(h)}(t), t \geq 0$. So it can be obtained from Eq. (1) by assuming $\beta(t)=\mu(t)=0$ for all $t$ (see [5] and the reference therein).

It is worth noting that beside the above mentioned particular cases, the Eq. (1) may be extended to vectorial case, i.e., when $X(t)$ is $\mathbb{R}^{d}-$ valued process, so other particular models can be deduced.

\section{THE SOLUTION PROCESSES OF COBL $(1,1)$}

Let $\mathfrak{J}^{(h)}=\mathfrak{I}\left(W^{(h)}\right):=\sigma\left(W^{(h)}(t), t \geq t_{0}\right)\left(\operatorname{resp} \mathfrak{\Im}_{t}^{(h)}:=\sigma\left(W^{(h)}(s), t_{0} \leq s \leq t\right)\right)$ be the $\sigma$-algebra generated by $\left(W^{(h)}(t)\right)_{t \geq 0}($ resp. generated by $W^{(h)}(s)$ up to time $t$ ) and let $\mathbb{L}_{2}\left(\mathfrak{J}^{(h)}\right)=\mathbb{L}_{2}\left(\mathbb{C}, \mathfrak{J}^{(h)}, P\right)$ (resp. $\mathbb{L}_{2}\left(\mathfrak{J}_{t}^{(h)}\right)$ ) be the Hilbert space of nonlinear $\mathbb{L}_{2}-$ functional of $\left(W^{(h)}(t)\right)_{t \geq 0}$. In this section, we are interested in solving the $S D E$ Eq. (1) in $\mathbb{L}_{2}\left(\mathfrak{\Im}_{t}^{(h)}\right)$. As already pointed by several authors (see for instance [6] for further discussions), that there is no general theory for the solution of $S D E$ driven by an $f B m$ if $h \neq 0$. Nevertheless, recently some studies was investigated the existence of such solutions for various families of $S D E$ driven by an $f B m$.

\subsection{The Itô Approach}

Our first approach is based on the Itô formula with respect to $f B m$ and the general results on $S D E$ to prove the uniqueness of the solution. First, we start by the fractional Itô's formula which is a powerful tool for dealing the solution. Consider the following SDE driven by $f B m$

$$
d X(t)=a(t, X(t)) d t+b(t, X(t)) d W^{h}(t), X\left(t_{0}\right)=X_{0}
$$

in which $a(.,),. b(.,$.$) are known continuous functions that represents the drift and diffusion respectively of the SDE Eq. (2) supposed to$ be smooth enough, and set $Y(t)=U(t, X(t))$ for some differentiable function $U: \mathbb{R} \rightarrow \mathbb{R}$. Then Dai and Heyde [7] have shown that the Itô formula with respect to $f B m$ is given by

$$
d Y(t)=\left\{\frac{\partial U}{\partial t}(t, X(t))+a(t, w) \frac{\partial U}{\partial x}(t, X(t))\right\} d t+b(t, w) \frac{\partial U}{\partial x}(t, X(t)) d W^{h}(t)
$$

Therefore, from the SDE Eq. (2) and the Itô formula Eq. (3) we obtain

$$
d Y(t)=\frac{\partial U}{\partial t}(t, X(t)) d t+\frac{\partial U}{\partial x}(t, X(t)) d X(t)
$$

So, the It $\hat{o}^{\prime} s$ solution of the SDE Eq. (1) is given by 
Theorem 2.1. Under the assumption 1, the unique Itô's solution of $S D E$ Eq. (1) in $\mathbb{L}_{2}\left(\mathfrak{J}^{(h)}\right)$ is given by

$$
X(t)=\Phi_{h}\left(t, t_{0}\right)\left\{X\left(t_{0}\right)+\int_{t_{0}}^{t} \Phi_{h}^{-1}\left(s, t_{0}\right) \mu(s) d s+\int_{t_{0}}^{t} \Phi_{h}^{-1}\left(s, t_{0}\right) \beta(s) d W^{h}(s)\right\}, t \geq t_{0}
$$

where $\Phi_{h}\left(t, t_{0}\right)=\exp \left\{\int_{t_{0}}^{t} \alpha(s) d s+\int_{t_{0}}^{t} \gamma(s) d W^{h}(s)\right\}$ with $\Phi_{h}\left(t_{0}, t_{0}\right)=1$ and the stochastic integral $\int_{t_{0}}^{t} \gamma(s) d W^{h}(s)$ is defined in Riemann's sense in probability.

Proof. First it is no difficult to see that $\Phi_{h}\left(t, t_{0}\right)$ is the unique solution of SDE

$$
d \Phi_{h}\left(t, t_{0}\right)=\alpha(t) \Phi_{h}\left(t, t_{0}\right) d t+\gamma(t) \Phi_{h}\left(t, t_{0}\right) d W^{h}(t)
$$

Now, set $\Phi_{h}\left(t, t_{0}\right)=\exp \{Y(t)\}, Z(t)=X(0)+\int_{t_{0}}^{t} e^{-Y(s)} \mu(s) d s+\int_{t_{0}}^{t} e^{-Y(s)} \beta(s) d W^{h}(s)$ and let $X(t)=U(Y(t), Z(t))$, where $U$ is the function defined by $U(x, y)=e^{x} y$. The fractional It $\hat{o}$ formula Eq. (3) and the expression Eq. (4) gives

$$
\begin{aligned}
d X(t) & =\frac{\partial U}{\partial x}(Y(t), Z(t)) d Y(t)+\frac{\partial U}{\partial y}(Y(t), Z(t)) d Z(t) \\
& =e^{Y(t)} Z(t) d Y(t)+e^{Y(t)} d Z(t) \\
& =X(t) d Y(t)+e^{Y(t)} d Z(t) \\
& =X(t)\left(\alpha(t) d t+\gamma(t) d W^{h}(t)\right)+e^{Y(t)}\left(e^{-Y(t)} \mu(t)+e^{-Y(t)} \beta(t) d W^{h}(t)\right) d t \\
& =(\alpha(t) X(t)+\mu(t)) d t+(\gamma(t) X(t)+\beta(t)) d W^{h}(t) .
\end{aligned}
$$

and hence the result follows.

Remark 1. If $\beta(t)=0$, then the Itô solution of $S D E$ Eq. (1) reduces to

$$
X(t)=\Phi_{h}\left(t, t_{0}\right)\left\{X\left(t_{0}\right)+\int_{t_{0}}^{t} \Phi_{h}^{-1}\left(s, t_{0}\right) \mu(s) d s\right\}, t \geq t_{0}
$$

and when $\gamma(t)=0$ and $\beta(t) \neq 0$, this is provides a solution of Gaussian $O U$ process, therefore if we are interested in non-Gaussian solution of Eq. (1), it is necessary to assume that $|\mu(t)|^{2}+|\beta(t)|^{2}>0$ and $\gamma(t) \neq 0$.

Remark 2. In time-invariant case, with $\mathfrak{R} e\{\gamma\}=0$ and $\mathfrak{R} e\{\alpha\}<0$, then the It $\hat{o}$ solution of $S D E$ Eq. (1) can be written as

$$
X(t)=\mu \int_{-\infty}^{t} \exp \left\{\alpha(t-s)+i \gamma\left(W^{h}(t)-W^{h}(s)\right)\right\} d s+\beta \int_{-\infty}^{t} \exp \{\alpha(t-s)\} d W^{h}(s)
$$

Remark 3. For any $t \geq t_{0}$, let $-\xi(t)=\int_{t_{0}}^{t} \alpha(s) d s+\int_{t_{0}}^{t} \gamma(s) d W^{h}(s)$ and $\eta^{h}(t)=\int_{t_{0}}^{t} \mu(s) d s+\int_{t_{0}}^{t} \beta(s) d W^{h}(s)$, then the solution process Eq. (5) may be rewritten as

$$
X(t)=e^{-\xi(t)}\left\{X\left(t_{0}\right)+\int_{t_{0}}^{t} e^{\xi(s)} d \eta^{h}(s)\right\}, t \geq t_{0}
$$

is the solution process of generalized Ornstein-Uhlenbeck (GOU) process driven by an $f B m$ defined by $d X(t)=-\xi(t) X(t) d t+d \eta^{h}(t), t \geq$ $t_{0}, X\left(t_{0}\right)=X_{0}$.

\subsection{The Frequency Approach}

In this subsection, we discuss a second approach to solve the SDE Eq. (1) based on the spectral representation. Indeed, it is now well known that for any regular second-order process $(X(t))_{t \geq t_{0}}$ (i.e., $X(t)$ is $\mathfrak{J}_{t}^{(h)}$-measurable not necessary stationary, belonging to $\left.\mathbb{L}_{2}\left(\mathfrak{J}^{(h)}\right)\right)$ admits the so-called Wiener-Itô (or Stratonovich) spectral representation, i.e.,

$$
X(t)=g_{t}(0)+\sum_{r \geq 1} \frac{1}{r !} \int_{\mathbb{R}^{r}} g_{t}\left(\underline{\lambda}_{(r)}\right) e^{i t \Sigma \underline{\lambda}_{(r)}} \prod_{j=1}^{r}\left(i \lambda_{j}\right)^{-h} d Z\left(\underline{\lambda}_{(r)}\right) .
$$


where $\underline{\lambda}_{(r)}=\left(\lambda_{1}, \ldots, \lambda_{r}\right), \Sigma \underline{\lambda}_{(r f)}=\sum_{i=1}^{r} \lambda_{i}$ and $d Z\left(\underline{\lambda}_{(r)}\right)=\prod_{j=1}^{r} d Z\left(\lambda_{i}\right)$ (see [8] for more details). The representation Eq. (8) is unique up to the permutation of the arguments of the evolutionary transfer functions $g_{t}\left(\underline{\lambda}_{(r)}\right), r \geq 2$ and $g_{t}\left(\underline{\lambda}_{(r)}\right) \in \mathbb{L}_{2}\left(G^{h}\right)=\mathbb{L}_{2}\left(\mathbb{C}^{n}, B_{\mathbb{C}^{n}}, G^{h}\right)$ for all $t \geq t_{0}$, with $d G^{h}\left(\lambda_{(r)}\right)=\frac{1}{(2 \pi)^{r}} \prod_{i=1}^{r}\left|\lambda_{i}\right|^{-2 h} d \lambda_{(r)}$ and such that

$$
\sum_{r \geq 0} \frac{1}{r !} \int_{\mathbb{R}^{r}}\left|g_{t}\left(\underline{\lambda}_{(r)}\right)\right|^{2} d G^{h}\left(\underline{\lambda}_{(r)}\right)<\infty \text { for all } t \geq t_{0} .
$$

Let us recall here the so-called the diagram formula for Wiener-Itô representation Eq. (8) which play an important role in some subsequent proofs and that state that for all $g$ and $f$ defined on $\mathbb{R}$ and on $\mathbb{R}^{r}$ respectively such that $(g, f) \in \mathbb{L}_{2}(\mathbb{R}) \times \mathbb{L}_{2 r}\left(\mathbb{R}^{r}\right)$, if $f$ is symmetric then

$$
\int_{\mathbb{R}} g(\lambda) d Z(\lambda) \int_{\mathbb{R}^{r}} f\left(\underline{\lambda}_{(r)}\right) d Z\left(\underline{\lambda}_{(r)}\right)=\int_{\mathbb{R}^{r+1}} g\left(\lambda_{r+1}\right) f\left(\underline{\lambda}_{(r)}\right) d Z\left(\underline{\lambda}_{(r+1)}\right)+\frac{r}{2 \pi} \int_{\mathbb{R}^{r-1}}\left\{\int \bar{g} \overline{\left.\mathbb{R}_{r}\right)} f\left(\underline{\lambda}_{(r)}\right) d \lambda_{r}\right\} d Z\left(\underline{\lambda}_{(r-1)}\right) .
$$

The spectral representation of the solution process of SDE Eq. (1) is given in the following theorem

Theorem 2.2. Assume that the process $(X(t))_{t \geq t_{0}}$ generated by the SDE Eq. (1) has a regular second-order solution. Then, the evolutionary symmetrized transfer functions $\left(\tilde{g}_{t}\left(\lambda_{(r)}\right)\right)_{t \geq t_{0}}, r \in \mathbb{N}$ of such solution are given by the symmetrization of the solution of the following first order ordinary differential equations

$$
g_{t}^{(1)}\left(\underline{\lambda}_{(r)}\right)=\left\{\begin{array}{l}
\alpha(t) g_{t}(0)+\mu(t)+\frac{\gamma(t)}{2 \pi} \int_{\mathbb{R}} g_{t}(\lambda)|\lambda|^{-2 h} d \lambda, r=0 \\
\left(\alpha(t)-i \Sigma \underline{\lambda}_{(r)}\right) g_{t}\left(\underline{\lambda}_{(r)}\right)+r \delta_{[r=1]} \beta(t) \\
+\gamma(t)\left(r g_{t}\left(\underline{\lambda}_{(r-1)}\right)+\frac{1}{2 \pi} \int_{\mathbb{R}} g_{t}\left(\underline{\lambda}_{(r+1)}\right)\left|\lambda_{r+1}\right|^{-2 h} d \lambda_{r+1}\right), r \geq 1
\end{array}\right.
$$

where the superscript ${ }^{(j)}$ denotes $j$-fold differentiation with respect to $t$ and where $\Sigma \underline{\lambda}_{(r)}=\sum_{i=1}^{r} \lambda_{i}$.

Proof. First, applying of the diagram formula for the nonlinear term $X(t) \frac{d W^{h}(t)}{d t}$ we get

$$
\begin{aligned}
& X(t) \frac{d W^{h}(t)}{d t}=\int_{\mathbb{R}} g_{t}(0) e^{i t \lambda}(i \lambda)^{-h} d Z(\lambda)+\sum_{r=1}^{\infty} \frac{1}{r !} \int_{\mathbb{R}^{r+1}} \tilde{g}_{t}\left(\underline{\lambda}_{(r)}\right) e^{i t \Sigma \underline{\lambda}_{(r+1)}} \prod_{t=1}^{r+1}\left(i \lambda_{l}\right)^{-h} d Z\left(\underline{\lambda}_{(r+1)}\right) \\
& +\sum_{r=1}^{\infty} \frac{1}{(r-1) !} \int_{\mathbb{R}^{r-1}} e^{i t \Sigma \underline{\lambda}_{(r-1)}}\left(\frac{1}{2 \pi} \int_{\mathbb{R}} g_{t}\left(\underline{\lambda}_{(r)}\right)\left|\lambda_{r}\right|^{-2 h} d \lambda_{r}\right) \prod_{l=1}^{r-1}\left(i \lambda_{l}\right)^{-h} d Z\left(\underline{\lambda}_{(r-1)}\right) .
\end{aligned}
$$

Second, we insert the spectral representation Eq. (8) of the process $(X(t))_{t \geq t_{0}}$ and the last expression of $X(t) d W^{h}(t)$ in the Eq. (1) the results follows.

Remark 4. The existence and uniqueness of the solution Eq. (10) is ensured by general results on linear ordinary differential equations, so

$$
g_{t}\left(\underline{\lambda}_{(r)}\right)=\left\{\begin{array}{l}
\varphi_{t}(0)\left(g_{t_{0}}(0)+\int_{t_{0}}^{t} \varphi_{s}^{-1}(0)\left(\mu(s)+\gamma(s) \frac{1}{2 \pi} \int_{\mathbb{R}} g_{s}(\lambda)|\lambda|^{-2 h} d \lambda\right) d s\right), r=0 \\
\varphi_{t}(\lambda)\left(g_{t_{0}}(\lambda)+\int_{t_{0}}^{t} \varphi_{s}^{-1}(\lambda)\left\{\beta(s)+\gamma(s)\left(g_{s}(0)+\frac{1}{2 \pi} \int_{\mathbb{R}} g_{s}\left(\underline{\lambda}_{(2)}\right)\left|\lambda_{2}\right|^{-2 h} d \lambda_{2}\right)\right\} d s\right), r=1 \\
\varphi_{t}\left(\underline{\lambda}_{(r)}\right)\left(g_{t_{0}}\left(\underline{\lambda}_{(r)}\right)+\int_{t_{0}}^{t} \varphi_{s}^{-1}\left(\underline{\lambda}_{(r)}\right) \gamma(s)\left(r g_{s}\left(\underline{\lambda}_{(r-1)}\right)+\frac{1}{2 \pi} \int_{\mathbb{R}} g_{s}\left(\underline{\lambda}_{(r+1)}\right)\left|\lambda_{r+1}\right|^{-2 h} d \lambda_{r+1}\right) d s\right), r \geq 2
\end{array}\right.
$$

in which $\varphi_{t}\left(\underline{\lambda}_{(r)}\right)=\exp \left\{\int_{t_{0}}^{t}\left(\alpha(s)-i \Sigma \underline{\lambda}_{(r)}\right) d s\right\}$.

Remark 5. Noting that beside the condition Eq. (9) a necessary conditions for that the evolutionary transfer functions $\left(g_{t}\left(\underline{\lambda}_{(r)}\right), r \in \mathbb{N}\right)$ defined by Eq. (11) determines a second-order process are

$$
\left.\left.\int_{\mathbb{R}}\left|\int_{\mathbb{R}} g_{t}\left(\underline{\lambda}_{(r+1)}\right)\right| \lambda_{r+1}\right|^{-2 h} d \lambda_{r+1}\right|^{2}\left|\lambda_{r}\right|^{-2 h} d \lambda_{r}<+\infty \text { and } \int_{\mathbb{R}}\left|g_{t}\left(\underline{\lambda}_{(r+1)}\right)\right|\left|\lambda_{r+1}\right|^{-2 h} d \lambda_{r+1}<+\infty
$$


for all $t \geq t_{0}$. These conditions are extremely difficult to be verified, except in time-invariant case when an explicit formula for the transfer functions are given (see for instance [9]).

It is worth noting that if $\mathfrak{R} e\{\gamma(t)\} \neq 0$, the $S D E$ Eq. (1) may be haven't a second-order solution, but it does if $\gamma(t)$ is purely imaginary. So in what follows, we consider the particular $S D E$

$$
d X(t)=(\alpha(t) X(t)+\mu(t)) d t+i \gamma(t) X(t) d W^{h}(t), t \geq t_{0}, X\left(t_{0}\right)=X_{0}
$$

and assume that

A3. $\alpha(t), \mu(t) \in \mathbb{C}, \gamma(t) \in \mathbb{R}$ and $\mathfrak{R} e\{\alpha(t)\}<0, \gamma(t) \neq 0$ for all $t \geq t_{0}$.

Under the condition A3, the Itô's solution of Eq. (12) reduces to

$$
X(t)=\Phi_{h}\left(t, t_{0}\right)\left\{X\left(t_{0}\right)+\int_{t_{0}}^{t} \Phi_{h}^{-1}\left(s, t_{0}\right) \mu(s) d s\right\}
$$

in which the function $\gamma(t)$ is replaced by $i \gamma(t)$. The spectral representation of Eq. (12) is given in the following lemma

Lemma 1. Assume that the process $(X(t))_{t \geq t_{0}}$ generated by the model Eq. (12) has a regular second-order solution. Then, the symmetrized evolutionary transfer functions $\left(\tilde{g}_{t}\left(\lambda_{(r)}\right)\right)_{t \in \mathbb{R}}, r \in \mathbb{N}$ of such solution may be obtained by the symmetrization of the following functions

$$
g_{t}\left(\lambda_{(r)}\right)=\left\{\begin{array}{l}
\varphi_{t}(0)\left(g_{t_{0}}(0)+\int_{t_{0}}^{t} \varphi_{s}^{-1}(0)\left(\mu(s)+i \gamma(s) \frac{1}{2 \pi} \int_{\mathbb{R}} g_{s}(\lambda)|\lambda|^{-2 h} d \lambda\right) d s\right), r=0 \\
\varphi_{t}\left(\underline{\lambda}_{(r)}\right)\left(g_{t_{0}}\left(\lambda_{(r)}\right)+i \int_{t_{0}}^{t} \varphi_{s}^{-1}\left(\underline{\lambda}_{(r)}\right) \gamma(s)\left(r g_{s}\left(\lambda_{(r-1)}\right)+\frac{1}{2 \pi} \int_{\mathbb{R}} g_{s}\left(\lambda_{(r+1)}\right)\left|\lambda_{r+1}\right|^{-2 h} d \lambda_{r+1}\right) d s\right), r \geq 1
\end{array}\right.
$$

Lemma 2. In time-invariant case we obtain

$$
g\left(\lambda_{(r)}\right)=\left\{\begin{array}{l}
g\left(\lambda_{(r)}\right)=-\frac{1}{\alpha}\left\{\mu+\frac{i \gamma}{2 \pi} \int_{\mathbb{R}} g(\lambda)|\lambda|^{-2 h} d \lambda\right\} \text { if } r=0 \\
\frac{-i \gamma}{\left(\alpha-i \underline{\lambda}_{(r)}\right)}\left\{r g\left(\lambda_{(r-1)}\right)+\frac{1}{2 \pi} \int_{\mathbb{R}} g\left(\lambda_{(r+1)}\right)\left|\lambda_{r+1}\right|^{-2 h} d \lambda_{r+1}\right\} \text { if } r \geq 1
\end{array}\right.
$$

so, its symmetrized version may be written as

$$
\tilde{g}\left(\lambda_{(r)}\right)=\operatorname{Sym}\left\{g\left(\lambda_{(r)}\right)\right\}=\mu(i \gamma)^{r} \int_{0}^{\infty} \exp \left\{\alpha u-\frac{\gamma^{2}}{2} k(h) u^{2 h+1}\right\} \prod_{j=1}^{r} \frac{1-e^{-i u \lambda_{j}}}{i \lambda_{j}} d u .
$$

\section{THE MOMENTS PROPERTIES AND THE SECOND-ORDER STRUCTURE}

In this section, we analyze the spectrum, i.e., the second-order structure of the process $(X(t))_{t \geq t_{0}}$ solution of the $S D E$ Eq. (1). For this purpose let $\left(\Psi_{h}\left(t, t_{0}\right)\right)_{t \geq t_{0}}$ be the mean function of the process $\left(\Phi_{h}\left(t, t_{0}\right)\right)_{t \geq t_{0}}$, and set $W_{h}(t, u, s, v)=h(2 h+1) \kappa(h) \int_{u}^{t} \int_{v}^{s} \gamma\left(v_{1}\right) \overline{\gamma\left(v_{2}\right)} \mid v_{1}-$ $\left.v_{2}\right|^{2 h-1} d v_{2} d v_{1}, u \leq t, v \leq s$. Then, we have

Lemma 3. Under the conditions of 1 , we have the following assertions

1. $\Psi_{h}\left(t, t_{0}\right)=\exp \left\{\int_{t_{0}}^{t} \alpha\left(v_{1}\right) d v_{1}+h(2 h+1) \frac{\kappa(h)}{2} \int_{t_{0}}^{t} \int_{t_{0}}^{t} \gamma\left(v_{1}\right) \gamma\left(v_{2}\right)\left|v_{1}-v_{2}\right|^{2 h-1} d v_{1} d v_{2}\right\}$ for $t \geq t_{0}$.

2. $E\left\{\Phi_{h}\left(t, t_{0}\right) \Phi_{h}^{-1}\left(u, t_{0}\right)\right\}=\Psi_{h}(t, u)$ for $t \geq u$.

3. $E\left\{\Phi_{h}\left(t, t_{0}\right) \overline{\Phi_{h}\left(s, t_{0}\right)}\right\}=\Psi_{h}\left(t, t_{0}\right) \overline{\Psi_{h}\left(s, t_{0}\right)} \exp \left\{W_{h}\left(t, t_{0}, s, t_{0}\right)\right\}$ for $t \geq s$.

4. $E\left\{\Phi_{h}\left(t, t_{0}\right) \overline{\Phi_{h}\left(s, t_{0}\right)} \overline{\Phi_{h}^{-1}\left(v, t_{0}\right)}\right\}=\Psi_{h}\left(t, t_{0}\right) \overline{\Psi_{h}(s, v)} \exp \left\{W_{h}\left(t, t_{0}, s, v\right)\right\}$ for $t \geq s \geq v$.

5. $E\left\{\Phi_{h}\left(t, t_{0}\right) \overline{\Phi_{h}\left(s, t_{0}\right)} \Phi_{h}^{-1}\left(u, t_{0}\right) \overline{\Phi_{h}^{-1}\left(v, t_{0}\right)}\right\}=\Psi_{h}(t, u) \overline{\Psi_{h}(s, v)} \exp \left\{W_{h}(t, u, s, v)\right\}$ for $t \geq s \geq v$. 
Proof. The assertions of the Lemma 3 follows upon observation that by using the expectation of exponential Gaussian process, we have

$$
\begin{aligned}
\Psi_{h}\left(t, t_{0}\right) & =\exp \left\{\int_{t_{0}}^{t} \alpha\left(v_{1}\right) d v_{1}+\frac{1}{2} E\left\{\left(\int_{t_{0}}^{t} \gamma\left(v_{1}\right) d W^{h}\left(v_{1}\right)\right)^{2}\right\}\right\} \\
& =\exp \left\{\int_{t_{0}}^{t} \alpha\left(v_{1}\right) d v_{1}+h(2 h+1) \frac{\kappa(h)}{2} \int_{t_{0}}^{t} \int_{t_{0}}^{t} \gamma\left(v_{1}\right) \gamma\left(v_{2}\right)\left|v_{1}-v_{2}\right|^{2 h-1} d v_{1} v_{2}\right\}
\end{aligned}
$$

and for $t \geq u$

$$
\begin{aligned}
E\left\{\Phi_{h}\left(t, t_{0}\right) \Phi_{h}^{-1}\left(u, t_{0}\right)\right\} & =\exp \left\{\int_{t_{0}}^{t} \alpha\left(v_{1}\right) d v_{1}+\frac{1}{2} E\left\{\left(\int_{t_{0}}^{t} \gamma\left(v_{1}\right) d W^{h}\left(v_{1}\right)\right)^{2}\right\}\right\} \\
& =\exp \left\{\int_{t_{0}}^{t} \alpha\left(v_{1}\right) d v_{1}+h(2 h+1) \frac{\kappa(h)}{2} \int_{u}^{t} \int_{u}^{t} \gamma\left(v_{1}\right) \gamma\left(v_{2}\right)\left|v_{1}-v_{2}\right|^{2 h-1} d v_{1} d v_{2}\right\} \\
& =\Psi_{h}(t, u) .
\end{aligned}
$$

and so on the rest are immediate.

Lemma 4. Under the condition of Lemma 3, the mean function $\left(m_{h}(t)=E\{X(t)\}\right)_{t \geq t_{0}}$ is given by

$$
m_{h}(t)=\Psi_{h}\left(t, t_{0}\right) m\left(t_{0}\right)+\int_{t_{0}}^{t} \Psi_{h}(t, u) \mu(u) d u, t \geq t_{0}
$$

and the covariance function $\left(R_{h}(t, s)=E\left\{\left(X(t)-m_{h}(t)\right) \overline{\left(X(s)-m_{h}(s)\right)}\right\}\right)_{t \geq s}$ is given by $R_{h}(t, s)=\Psi_{h}\left(t, t_{0}\right) \overline{\Psi_{h}\left(s, t_{0}\right)} \exp \left\{W_{h}\left(t, t_{0}, s, t_{0}\right)\right\} R\left(t_{0}\right)+\left.\Psi_{h}\left(t, t_{0}\right) \overline{\Psi_{h}\left(s, t_{0}\right)}\left[\exp \left\{W_{h}\left(t, t_{0}, s, t_{0}\right)\right\}-1\right] \operatorname{Im}\left(t_{0}\right)\right|^{2}$

$$
\begin{aligned}
& +m\left(t_{0}\right) \int_{t_{0}}^{s} \Psi_{h}\left(t, t_{0}\right) \overline{\Psi_{h}(s, v)}\left[\exp \left\{W_{h}\left(t, t_{0}, s, v\right)\right\}-1\right] \overline{\mu(v)} d v \\
& +\overline{m\left(t_{0}\right)} \int_{t_{0}}^{t} \overline{\Psi_{h}\left(s, t_{0}\right)} \Psi_{h}(t, u)\left[\exp \left\{W_{h}\left(t, u, s, t_{0}\right)\right\}-1\right] \mu(u) d u \\
& +\int_{t_{0}}^{t} \int_{t_{0}}^{s} \Psi_{h}(t, u) \overline{\Psi_{h}(s, v)}\left[\exp \left\{W_{h}(t, u, s, v)\right\}-1\right] \overline{\mu(v)} \mu(u) d v d u \\
& +h(2 h+1) \kappa(h) \int_{t_{0}}^{t} \int_{t_{0}}^{s} \Psi_{h}(t, u) \overline{\Psi_{h}(s, v)} \exp \left\{W_{h}(t, u, s, v)\right\} \overline{\beta(v)} \beta(u)|u-v|^{2 h-1} d v d u
\end{aligned}
$$

Proof. From the It ô's solution Eq. (5), we can obtain

$$
\begin{aligned}
m_{h}(t) & =E\{X(t)\}=E\left\{\Phi_{h}\left(t, t_{0}\right) X\left(t_{0}\right)\right\}+\int_{t_{0}}^{t} E\left\{\Phi_{h}\left(t, t_{0}\right) \Phi_{h}^{-1}\left(u, t_{0}\right)\right\} \mu(u) d u \\
& =\Psi_{h}\left(t, t_{0}\right) m\left(t_{0}\right)+\int_{t_{0}}^{t} \Psi_{h}(t, u) \mu(u) d u
\end{aligned}
$$


Since $W^{h}(t)$ independent of $X\left(t_{0}\right)$, then $E\left\{\Phi_{h}\left(t, t_{0}\right) X\left(t_{0}\right)\right\}=E\left\{\Phi_{h}\left(t, t_{0}\right)\right\} E\left\{X\left(t_{0}\right)\right\}=\Psi_{h}\left(t, t_{0}\right) m_{h}\left(t_{0}\right)$. In order to evaluate the expression of $R_{h}(t, s)$ we use the Itô's solution Eq. (5) to obtain

$$
\begin{aligned}
E\{X(t) \overline{X(s)}\} & =E\left\{\Phi_{h}\left(t, t_{0}\right) \overline{\Phi_{h}\left(s, t_{0}\right)}\right\} E\left\{\left|X\left(t_{0}\right)\right|^{2}\right\}+m\left(t_{0}\right) \int_{t_{0}}^{s} E\left\{\Phi_{h}\left(t, t_{0}\right) \overline{\Phi_{h}\left(s, t_{0}\right)} \overline{\Phi_{h}^{-1}\left(v, t_{0}\right)}\right\} \overline{\mu(v)} d v \\
& +\overline{m\left(t_{0}\right)} \int_{t_{0}}^{t} E\left\{\overline{\Phi_{h}\left(s, t_{0}\right)} \Phi_{h}\left(t, t_{0}\right) \Phi_{h}^{-1}\left(u, t_{0}\right)\right\} \mu(u) d u \\
& +\int_{t_{0}}^{t} \int_{t_{0}}^{s} E\left\{\Phi_{h}\left(t, t_{0}\right) \overline{\Phi_{h}\left(s, t_{0}\right)} \Phi_{h}^{-1}\left(u, t_{0}\right) \overline{\Phi_{h}^{-1}\left(v, t_{0}\right)}\right\} \overline{\mu(v)} \mu(u) d v d u \\
& +h(2 h+1) \kappa(h) \int_{t_{0}}^{t} \int_{t_{0}}^{s} E\left\{\Phi_{h}\left(t, t_{0}\right) \overline{\Phi_{h}\left(s, t_{0}\right)} \Phi_{h}^{-1}\left(u, t_{0}\right) \overline{\Phi_{h}^{-1}\left(v, t_{0}\right)}\right\} \overline{\beta(v)} \beta(u)|u-v|^{2 h-1} d v d u,
\end{aligned}
$$

In other hand

$$
\begin{aligned}
m_{h}(t) \overline{m_{h}(s)} & =\Psi_{h}\left(t, t_{0}\right) \overline{\Psi_{h}\left(s, t_{0}\right)}\left|m\left(t_{0}\right)\right|^{2}+m\left(t_{0}\right) \int_{t_{0}}^{s} \Psi_{h}\left(t, t_{0}\right) \overline{\Psi_{h}(s, v) \mu(v)} d v \\
& +\overline{m\left(t_{0}\right)} \int_{t_{0}}^{t} \overline{\Psi_{h}\left(s, t_{0}\right)} \Psi_{h}(s, u) \mu(u) d u+\int_{t_{0}}^{s} \int_{t_{0}}^{s} \Psi_{h}(t, u) \overline{\Psi_{h}(s, v) \mu(v)} \mu(u) d v d u
\end{aligned}
$$

the fact that $R_{h}(t, s)=E\{X(t) \overline{X(s)}\}-m_{h}(t) \overline{m_{h}(s)}$ the expression for $R_{h}(t, s)$ follows.

Lemma 5. Consider the time-invariant process $(X(t))_{t \geq t_{0}}$ generated bySDE Eq. (1). Then under the condition 1 , the mean and covariance functions of the solution process $(X(t))_{t \geq t_{0}}$ are given by

$$
\begin{aligned}
& m_{h}=\mu \int_{0}^{\infty} K_{h}(u) d u \\
& R_{h}(|\tau|)=|\mu|^{2} \int_{0}^{\infty} \int_{0}^{\infty} K_{h}\left(u_{1}\right) \overline{K_{h}\left(u_{2}\right)}\left(\exp \left\{-\frac{\gamma^{2}}{2} \kappa(h) W_{(\tau)}^{h}\left(u_{1}, u_{2}\right)\right\}-1\right) d u_{1} d u_{2} \\
& +|\beta|^{2} h(2 h+1) \kappa(h) \int_{0}^{\infty} \int_{0}^{\infty} K_{h}\left(u_{1}\right) \overline{K_{h}\left(u_{2}\right)} \exp \left\{-\frac{\gamma^{2}}{2} \kappa(h) W_{(\tau)}^{h}\left(u_{1}, u_{2}\right)\right\} d u_{1} d u_{2},
\end{aligned}
$$

where

$$
W_{(\tau)}^{h}\left(u_{1}, u_{2}\right)=|\tau|^{2 h+1}-\left|\tau-u_{1}\right|^{2 h+1}-\left|\tau+u_{2}\right|^{2 h+1}+\left|\tau-u_{1}+u_{2}\right|^{2 h+1},
$$

and $K_{h}(t)=\exp \left\{\alpha t-\frac{\gamma^{2}}{2} \kappa(h) t^{2 h+1}\right\}$.

Proof. Straightforward and hence omitted.

Corollary 1. Consider the time-invariant version of the SDE Eq. (12), then $\lim _{\tau \rightarrow+\infty} \frac{R(\tau)}{c \tau^{-\delta}}=1$ for some constant $c$ and $0<\delta<1$, this means that the solution process exhibits long range dependence. In this case the dependence between $X(t)$ and $X(t+\tau)$ decays slowly as $\tau \rightarrow+\infty$ and. $\int_{\mathbb{R}} R(|\tau|) d \tau=\infty$.

Proof. First we have

$$
\exp \left\{-\frac{\gamma^{2}}{2} \kappa(h)\left(|\tau|^{2 h+1}-\left|\tau-u_{1}\right|^{2 h+1}-\left|\tau+u_{2}\right|^{2 h+1}+\left|\tau-u_{1}+u_{2}\right|^{2 h+1}\right)\right\}
$$




$$
=\exp \left\{-\frac{\gamma^{2}}{2} \kappa(h)|\tau|^{2 h+1}\left(1-\left|1-\frac{u_{1}}{\tau}\right|^{2 h+1}-\left|1+\frac{u_{2}}{\tau}\right|^{2 h+1}+\left|1+\frac{u_{2}-u_{1}}{\tau}\right|^{2 h+1}\right)\right\}
$$

and

$$
\begin{gathered}
\left(1-\frac{u_{1}}{\tau}\right)^{2 h+1}=1-(2 h+1) \frac{u_{1}}{\tau}+\frac{(2 h+1)(2 h)}{2} \frac{u_{1}^{2}}{\tau^{2}}+\ldots \tau \rightarrow+\infty \\
\left(1+\frac{u_{2}}{\tau}\right)^{2 h+1}=1+(2 h+1) \frac{u_{2}}{\tau}+\frac{(2 h+1)(2 h)}{2} \frac{u_{2}^{2}}{\tau^{2}}+\ldots \tau \rightarrow+\infty \\
\left(1+\frac{u_{2}-u_{1}}{\tau}\right)^{2 h+1}=1+(2 h+1) \frac{\left(u_{2}-u_{1}\right)}{\tau}+\frac{(2 h+1)(2 h)}{2} \frac{\left(u_{2}-u_{1}\right)^{2}}{\tau^{2}}+\ldots \tau \rightarrow+\infty .
\end{gathered}
$$

Let $\delta=-(2 h-1)$, it is clear $0<\delta<1$ because $0<h<\frac{1}{2}$, then we have

$$
\lim _{\tau \rightarrow+\infty} \frac{\exp \left\{-\frac{\gamma^{2}}{2} \kappa(h) W_{\tau}^{h}\left(u_{1}, u_{2}\right)\right\}-1}{\tau^{-\delta}}=\lim _{\tau \rightarrow+\infty} \frac{\exp \left\{\frac{\gamma^{2}}{2} \kappa(h) h(2 h+1) u_{1} u_{2} \tau^{2 h-1}\right\}-1}{\tau^{2 h-1}}=\frac{\gamma^{2}}{2} h(2 h+1) u_{1} u_{2} .
$$

It follows that

$$
\begin{aligned}
\lim _{\tau \rightarrow+\infty} \frac{R(\tau)}{\tau^{-\delta}} & =|\mu|^{2} \int_{0}^{\infty} \int_{0}^{\infty} K_{h}\left(u_{1}\right) \overline{K_{h}\left(u_{2}\right)} \lim _{\tau \rightarrow+\infty} \tau^{\delta}\left\{\exp \left\{-\frac{\gamma^{2}}{2} \kappa(h) W_{\tau}^{h}\left(u_{1}, u_{2}\right)\right\}-1\right\} d u_{1} d u_{2} \\
& =\frac{\gamma^{2}}{2} \kappa(h) h(2 h+1)|\mu|^{2} \int_{0}^{\infty} u_{1} K_{h}\left(u_{1}\right) d u_{1} \int_{0}^{\infty} u_{1} K_{h}\left(u_{2}\right) d u_{2} \\
& =\frac{\gamma^{2}}{2} \kappa(h) h(2 h+1)|\mu|^{2}\left|\int_{0}^{\infty} u K_{h}(u) d u\right|^{2}=c<\infty,
\end{aligned}
$$

Hence, the process $(X(t))_{t \geq 0}$ generated by the $S D E$ Eq. (12) with time-invariant parameters is a long memory process.

\subsection{Third-Order Structure of COBL(1,1) Process}

For the sake of convenience and simplicity, we shall consider the time-invariant version of the SDE Eq. (1). Moreover, we assume the process solution admits the spectral representation Eq. (8) in which the symmetrized version of transfer functions $g\left(\underline{\lambda}_{(r)}\right)$ may be written as

$$
g\left(\lambda_{(r)}\right)=\mu(i \gamma)^{r} \int_{0}^{\infty} K_{h}(u) \frac{1-e^{-i u \lambda_{j}}}{i \lambda_{j}} d u, \forall r \geq 0 .
$$

Then using the representation Eq. (8) we can obtain the following approximation

$$
\begin{aligned}
X(t) & =g(0)+\int_{\mathbb{R}} g\left(\lambda_{1}\right) e^{i t \lambda_{1}} d Z\left(\lambda_{1}\right)+\int_{\mathbb{R}^{2}} g\left(\lambda_{(2)}\right) e^{i t \lambda_{(2)}} d Z\left(\lambda_{(2)}\right)+\xi(t) \\
& =X^{(1)}(t)+X^{(2)}(t)+\xi(t),
\end{aligned}
$$

where $\xi(t)$ is a second-order stationary process which it is orthogonal to the first two terms. The symmetrized transfer functions $\tilde{g}\left(\lambda_{1}\right)$ and $\tilde{g}\left(\lambda_{(2)}\right)$ are given by

$$
g\left(\lambda_{1}\right)=\mu(i \gamma) \int_{0}^{\infty} K_{h}(u) \frac{1-e^{-i u \lambda_{1}}}{i \lambda_{1}} d u \text { and } g\left(\lambda_{1}, \lambda_{2}\right)=\mu(i \gamma)^{2} \int_{0}^{\infty} K_{h}(u) \prod_{j=1}^{2} \frac{1-e^{-i u \lambda_{2}}}{i \lambda_{2}} d u
$$

It can be shown that

$$
\begin{aligned}
& C_{h}(s, u)=E\{(X(t)-g(0))((X(t+s)-g(0))((X(t+u)-g(0))\} \\
& =E\left\{X^{(1)}(t) X^{(1)}(t+s) X^{(2)}(t+u)\right\}+E\left\{X^{(1)}(t) X^{(2)}(t+s) X^{(1)}(t+u)\right\} \\
& +E\left\{X^{(2)}(t) X^{(1)}(t+s) X^{(1)}(t+u)\right\}+O(1) .
\end{aligned}
$$


We calculate $E\left\{X^{(1)}(t) X^{(1)}(t+s) X^{(2)}(t+u)\right\}$, and the other terms can be obtained by symmetry. First we observe that $E\left\{X^{(1)}(t) X^{(1)}(t+s) X^{(2)}(t+u)\right\}$

$=E\left\{\int_{\mathbb{R}^{2}} g\left(\lambda_{1}\right) g\left(\lambda_{2}\right) e^{i t \lambda_{1}+i(t+s) \lambda_{2}} d Z\left(\lambda_{(2)}\right) \int_{\mathbb{R}^{2}} g\left(\lambda_{3}, \lambda_{4}\right) e^{i(t+u)\left(\lambda_{3}+\lambda_{4}\right)} d Z\left(\lambda_{3}, \lambda_{4}\right)\right\}$

$=2 ! \int_{\mathbb{R}^{2}} \operatorname{sym}\left\{g\left(\lambda_{1}\right) g\left(\lambda_{2}\right) e^{i t \lambda_{1}+i(t+s) \lambda_{2}}\right\} \overline{\operatorname{sym}\left\{g\left(\lambda_{1}, \lambda_{2}\right) e^{i(t+u)\left(\lambda_{1}+\lambda_{2}\right)}\right\}} d F\left(\lambda_{(2)}\right)$

$=2 \int_{\mathbb{R}^{2}} g\left(\lambda_{1}\right) g\left(\lambda_{2}\right) g\left(-\lambda_{1},-\lambda_{2}\right) \operatorname{sym}\left\{e^{i s \lambda_{1}}\right\} e^{-i u\left(\lambda_{1}+\lambda_{2}\right)} \frac{d \lambda_{1} \lambda_{2}}{(2 \pi)^{2}}$

$=\frac{1}{(2 \pi)^{2}}\left\{\int_{\mathbb{R}^{2}} g\left(\lambda_{1}\right) g\left(\lambda_{2}\right) g\left(-\lambda_{1},-\lambda_{2}\right) e^{i s \lambda_{1}} e^{-i u\left(\lambda_{1}+\lambda_{2}\right)} d \lambda_{1} \lambda_{2}+\int_{\mathbb{R}^{2}} g\left(\lambda_{1}\right) g\left(\lambda_{2}\right) g\left(-\lambda_{1},-\lambda_{2}\right) e^{i s \lambda_{2}} e^{-i u\left(\lambda_{1}+\lambda_{2}\right)} d \lambda_{1} \lambda_{2}\right\}$

$=\frac{1}{(2 \pi)^{2}}\left\{\int_{\mathbb{R}^{2}} g\left(\lambda_{1}\right) g\left(\lambda_{2}\right) g\left(-\lambda_{1},-\lambda_{2}\right) e^{i(s-u) \lambda_{1}-i u \lambda_{2}} d \lambda_{1} \lambda_{2}+\int_{\mathbb{R}^{2}} g\left(\lambda_{1}\right) g\left(\lambda_{2}\right) g\left(-\lambda_{1},-\lambda_{2}\right) e^{-i u \lambda_{1}+i(s-u) \lambda_{2}} d \lambda_{1} \lambda_{2}\right\}$.

Moreover we have

$$
\begin{aligned}
& E\left\{X^{(1)}(t) X^{(2)}(t+s) X^{(1)}(t+u)\right\} \\
& =E\left\{\int_{\mathbb{R}^{2}} g\left(\lambda_{1}\right) g\left(\lambda_{2}\right) e^{i t \lambda_{1}+i(t+u) \lambda_{2}} d Z\left(\lambda_{(2)}\right) \int_{\mathbb{R}^{2}} g\left(\lambda_{3}, \lambda_{4}\right) e^{i(t+s)\left(\lambda_{3}+\lambda_{4}\right)} d Z\left(\lambda_{3}, \lambda_{4}\right)\right\} \\
& =2 ! \int_{\mathbb{R}^{2}} \operatorname{sym}\left\{g\left(\lambda_{1}\right) g\left(\lambda_{2}\right) e^{i t \lambda_{1}+i(t+u) \lambda_{2}}\right\} \overline{\operatorname{sym}\left\{g\left(\lambda_{1}, \lambda_{2}\right) e^{i(t+s)\left(\lambda_{1}+\lambda_{2}\right)}\right\}} d F\left(\lambda_{(2)}\right) \\
& =2 \int_{\mathbb{R}^{2}} g\left(\lambda_{1}\right) g\left(\lambda_{2}\right) g\left(-\lambda_{1},-\lambda_{2}\right) \operatorname{sym}\left\{e^{i u \lambda_{1}}\right\} e^{-i s\left(\lambda_{1}+\lambda_{2}\right)} \frac{d \lambda_{1} \lambda_{2}}{(2 \pi)^{2}} \\
& =\frac{1}{(2 \pi)^{2}}\left\{\int_{\mathbb{R}^{2}} g\left(\lambda_{1}\right) g\left(\lambda_{2}\right) g\left(-\lambda_{1},-\lambda_{2}\right) e^{i u \lambda_{1}} e^{-i s\left(\lambda_{1}+\lambda_{2}\right)} d \lambda_{1} \lambda_{2}+\int_{\mathbb{R}^{2}} g\left(\lambda_{1}\right) g\left(\lambda_{2}\right) g\left(-\lambda_{1},-\lambda_{2}\right) e^{i u \lambda_{2}} e^{-i s\left(\lambda_{1}+\lambda_{2}\right)} d \lambda_{1} \lambda_{2}\right\} \\
& =\frac{1}{(2 \pi)^{2}}\left\{\int_{\mathbb{R}^{2}} g\left(\lambda_{1}\right) g\left(\lambda_{2}\right) g\left(-\lambda_{1},-\lambda_{2}\right) e^{i(u-s) \lambda_{1}-i s \lambda_{2}} d \lambda_{1} \lambda_{2}+\int_{\mathbb{R}^{2}} g\left(\lambda_{1}\right) g\left(\lambda_{2}\right) g\left(-\lambda_{1},-\lambda_{2}\right) e^{-i s \lambda_{1}+i(u-s) \lambda_{2}} d \lambda_{1} \lambda_{2}\right\} .
\end{aligned}
$$

It remains to compute $E\left\{X^{(2)}(t) X^{(1)}(t+s) X^{(1)}(t+u)\right\}$, then

$$
\begin{aligned}
& E\left\{X^{(2)}(t) X^{(1)}(t+s) X^{(1)}(t+u)\right\} \\
& =E\left\{\int_{\mathbb{R}^{2}} g\left(\lambda_{3}\right) g\left(\lambda_{4}\right) e^{i(t+s) \lambda_{3}+i(t+u) \lambda_{4}} Z\left(d \lambda_{3}, d \lambda_{4}\right) \int_{\mathbb{R}^{2}} g\left(\lambda_{1}, \lambda_{2}\right) e^{i t\left(\lambda_{1}+\lambda_{2}\right)} Z\left(d \lambda_{(2)}\right)\right\} \\
& =2 ! \int_{\mathbb{R}^{2}} \operatorname{sym}\left\{g\left(\lambda_{1}\right) g\left(\lambda_{2}\right) e^{i(t+s) \lambda_{1}+i(t+u) \lambda_{2}}\right\} \frac{\operatorname{sym}\left\{g\left(\lambda_{1}, \lambda_{2}\right) e^{i t\left(\lambda_{1}+\lambda_{2}\right)}\right\}}{\operatorname{sy}} d F\left(\lambda_{(2)}\right) \\
& \left.=2 \int_{\mathbb{R}^{2}} g\left(\lambda_{1}\right) g\left(\lambda_{2}\right) g\left(-\lambda_{1},-\lambda_{2}\right)\right) \operatorname{sym}\left\{e^{i s \lambda_{1}+i u \lambda_{2}}\right\} \frac{d \lambda_{1} \lambda_{2}}{(2 \pi)^{2}} \\
& =\frac{1}{(2 \pi)^{2}}\left\{\int_{\mathbb{R}^{2}} g\left(\lambda_{1}\right) g\left(\lambda_{2}\right) g\left(-\lambda_{1},-\lambda_{2}\right) e^{i s \lambda_{1}+i u \lambda_{2}} d \lambda_{1} \lambda_{2}+\int_{\mathbb{R}^{2}} g\left(\lambda_{1}\right) g\left(\lambda_{2}\right) g\left(-\lambda_{1},-\lambda_{2}\right) e^{i u \lambda_{1}+i s \lambda_{2}} d \lambda_{1} \lambda_{2}\right\} .
\end{aligned}
$$


Hence

$$
C_{h}(s, u)=2 \int_{\mathbb{R}^{2}} g\left(\lambda_{1}\right) g\left(\lambda_{2}\right) g\left(-\lambda_{1},-\lambda_{2}\right) \operatorname{sym}\left\{e^{i(s-u) \lambda_{1}-u \lambda_{2}}+e^{e^{i(u-s) \lambda_{1}-s \lambda_{2}}}+e^{i\left(s \lambda_{1}+u \lambda_{2}\right)}\right\} \frac{d \lambda_{1} \lambda_{2}}{(2 \pi)^{2}} .
$$

By taking Fourier transforms (omitting the terms of $O(1)$ ), the bispectral density function $f\left(\lambda_{1}, \lambda_{2}\right)$ can be shown to be $f\left(\lambda_{1}, \lambda_{2}\right)=$ $\frac{2}{(2 \pi)^{2}}\left\{S\left(\lambda_{1}, \lambda_{2}\right)+S\left(\lambda_{2},-\lambda_{1}-\lambda_{2}\right)+S\left(\lambda_{1},-\lambda_{1}-\lambda_{2}\right)\right\}$ where $S\left(\lambda_{1}, \lambda_{2}\right)=g\left(\lambda_{1}\right) g\left(\lambda_{2}\right) g\left(-\lambda_{1},-\lambda_{2}\right)$. It is clear from the above that the bispectrum is zero for all frequencies $\lambda_{1}$ and $\lambda_{2}$ if and only if the process is linear $(\gamma=0)$ (and Gaussian).

\section{CONCLUSION}

This paper describes some basic probabilistic properties of COBL process driven by an $(f) B m$. Our main aim was focused firstly on the existence of the solution in time-frequency domain and secondary to prove that the use of $f B m$ as innovation we led to a long-range dependency property.

\section{COMPETING INTEREST}

Stochastic differential equation $(S D E)$, Estimation of $S D E$, Asymptotic Inference.

\section{ACKNOWLEDGMENT}

We would like to thank Prof. M. Ahsanullah Editor-in-Chief of the journal, for his attention, encouragement and valuable advice. Also, we are very grateful to an anonymous referee for reading the paper very carefully and making many constructive remarks and suggestions.

\section{REFERENCES}

1. Y. S. Mishura, Stochastic calculus for fractional Brownian motion and related processes, Lecture Notes in Mathematics, vol. 1929, SpringerVerlag, Berlin, 2008.

2. T. E. Duncan, B. Pasik-Duncan, Parameter identification for some linear systems with fractional Brownian motion, 15th Triennial World Congress, Barcelona, IFAC Proceedings, vol. 35(1) (2002), 319-324.

3. G. Shen, X. Yin, L. Yan, Acta Mathematica Scientia, 36B(2) 2016, 394-408.

4. A. Swishchuk, Modeling and Pricing of Swaps for Financial and Energy Markets with Stochastic Volatilities, World Scientific Books, World Scientific Publishing Co. Pte. Ltd, Singapore, 2013.

5. C. Bendr, P. Parczewski, Bernoulli, 16(2) (2010), 389-417.

6. T. E. Duncan, in: Stochastic Processes, Optimization, and Control Theory: Applications in Financial Engineering, Queueing Networks, and Manufacturing Systems, Springer, Boston, 2006, pp. 97-108.

7. W. Dai, C.C. Heyde, J. Appl. Math. Stoch. Anal. 9(4) (1996), 439-448.

8. A. Bibi, F. Merahi, Int. J. Stat. Probab. 4(3) (2015), 150-160.

9. E. Iglói, G. Terdik, Ann. Appl. Probab. 9(1) (1999), 46-77. 